\title{
Medical Image Registration Based on Inertia Matrix and Iterative Closest Point
}

\author{
Mei-sen Pan, Jian-jun Jiang, Qiu-sheng Rong and Fen Zhang \\ College of Computer Science and Technology, Hunan University of arts \& \\ sciences, Changde 415000 China \\ pmsjjj@126.com
}

\begin{abstract}
The closest iterative point algorithm (ICP) is widely used in medical image registration. But there exist some problems in the following aspects. First, due to its heavily computational load, it has a time-consuming process and a low registration efficiency. Second, due to the fact that it heavily depends on whether the initial rotation and translation matrices of the floating point set can be exactly extracted, it often traps in the local optimum and even fails to register images. In addition, due to the complexity of medical images, it is difficult to automatically extract the salutary feature points. In this paper, by computing the coordinate inertia matrices of the reference and floating images, the rotation angles are obtained and referred to as the initial rotation parameters of ICP for image registration. The edges of the reference and floating images are detected by the edge convolution kernel so-called B-spline gradient operator (BSGO) and then the binarization images involving the feature points are acquired. The experimental results reveal that, this proposed method has a fairly simple implementation, a low computational load, a fast registration and good alignment accuracy. Also, It can efficiently avoid trapping in the local optimum and cater to both mono-modality and multi-modality image registrations.
\end{abstract}

Keywords: Medical image; Image registration; Inertia matrix; Iterative closest point

\section{Introduction}

For the past few years, in the wake of the development of imaging technology, the imaging quality and image resolution generated by various imaging devices such as Computerized Tomography (CT), Magnetic Resonance Imaging (MRI), Single-Photon Emission Computed Tomography (SPECT), Positron emission computed tomography (PET) and so on, have been greatly raised and provide the credible evidence for accurate clinical diagnosis and treatment. However, due to the far different imaging principle among various imaging devices and the diverse image information acquired by them, the research about aligning the medical images generated by various imaging devices, becomes a hot field which has been attracting increasing attention around the world over the years.

Medical image registration, as the prerequisite for medical image fusion, signifies that the spatial geometric transformation is applied to match two or more images produced by various imaging devices, and enables the pixels (voxels) representing the same structure to achieve the space correspondence [1-2]. In the past several decades, medical image registration methods have achieved rapid development, and researchers all over the world have proposed many practical and effective technologies. In general, these methods are grouped into two categories: intensity-based and feature-based medical image registration methods [3].

With respect to the intensity-based image registration, it only uses the gray level similarity between two images as the registration function. Due to the full use of image 
gray information, it has fairly higher registration accuracy. The mutual information (MI)based registration method, as an intensity-based one, has become one of the most extensively-used methods because it is more flexible and accurate than any other methods in the global information context [4-5]. In the process of the image registration, it employs MI as similarity function, and can automatically and accurately align two or more images without any prior segmentation or other preprocessing. The MI method, however, has the following native defect. First, due to the fact that it only covers the gray information with less or no consideration of spatial information, it often traps in the local optimum and even fails to align images. Moreover, it is a massively computational load and low registration efficiency.

For the feature-based image registration, it extracts the common, distinct and significant features between the registering images to explore the transformation parameters. It is effective and easy to implement, but its registration accuracy seriously depends on whether the image feature points can be exactly extracted [6-7]. In consideration of the complexity of various medical images, it is difficult to automatically and accurately abstract and refine the useful feature points from medical images. Therefore, its poor adaptability still needs to be further raised. The iterative closest point algorithm (ICP), as a feature-based registration method pioneered by Besl and Mckay [8] in 1992, is one well known approach and extensively used for the registration on point sets. It can align the point sets without any prior and explicit segmentation or other preprocessing of object features, which makes it to be prevalent and very well adapted for point set registration [2].

In this paper, on the foundation of a comprehensive and thorough research into image coordinate inertia matrix and ICP, we propose medical image registration based on inertia matrix and iterative closest point (IMICP). First, the centroids of the reference and floating images are computed by using the image moments to obtain the initial translation values along $\mathrm{X}, \mathrm{Y}$ directions, and the rotation angles are extracted by using the coordinate inertia matrices of the reference and floating images to compute the initial rotation parameters. So far, the initial parameters for registration of IMICP have been obtained. Second, the binarization images are acquired after the B-spline gradient operator (BSGO) [2] is employed to probe the edges of the reference and floating images, which will be selected as the feature points in the process of IMICP, namely, the reference and floating point sets for registration are acquired. Finally, ICP is applied to explore the optimal transformation parameters. This proposed method can cater to both mono-modality and multi-modality image registrations.

\section{Defect of the Conventional Feature-based Image Registration}

In 1980's, the researchers around the world made deep and systematic study on the registration of point sets. In particular, Arun et al. [9] introduced an approach to alignment between point sets using unit quaternions to represent rotation in 1987. Quite a number of experiments and practices have showed that the alignment technology is an efficient way to tackle the intractable issues of registration. In 1992, Besl and Mckay [8], as researchers in the field of computer vision, pioneered ICP to deal with the issue of point alignment. Since ICP can align the point sets without any prior and explicit segmentation or other preprocessing of object features, it becomes very important and is very propitious to align point sets. Therefore, it is widely used in various alignment fields.

Suppose that two point sets $\boldsymbol{X}, \boldsymbol{Y} \in R^{K}, \boldsymbol{X}=\left\{\boldsymbol{x}_{i}, i=1,2, \cdots, N\right\}$ with $\boldsymbol{x}_{i}=\left[\begin{array}{lll}x_{i 1} & \cdots & x_{i K}\end{array}\right]^{T}$ and $\boldsymbol{Y}=\left\{\boldsymbol{y}_{j}, j=1,2, \cdots, M\right\}$ with $\boldsymbol{y}_{j}=\left[\begin{array}{lll}y_{j 1} & \cdots & y_{j K}\end{array}\right]^{T}$ present the reference and floating point sets respectively, and $\boldsymbol{Z}=\left\{z_{i}, i=1,2, \cdots, M\right\}$ with $z_{i}=\left[\begin{array}{lll}z_{i 1} & \cdots & z_{i K}\end{array}\right]^{T}$ and $z_{i} \in \boldsymbol{X}$ expresses the closest point set. In addition, $\boldsymbol{R}_{0}$ and $\boldsymbol{T}_{0}$ denote $K \times K$ rotation and $K \times 1$ translation matrices respectively. The aim of ICP proposed by Besl and Mckay [8] is to 
explore a rigid transformation $\left(\boldsymbol{R}_{0}, \boldsymbol{T}_{0}\right)$ to minimize the mean square sum of the Euclidean distances between the floating point set $\boldsymbol{Y}$ transformed by $\left(\boldsymbol{R}_{0}, \boldsymbol{T}_{0}\right)$ and its closest point set $\boldsymbol{Z}$ in $\boldsymbol{X}$, namely, the objection function to be minimized is

$$
J\left(\boldsymbol{R}_{0}, \boldsymbol{T}_{0}\right)=\operatorname{Min}\left\{\frac{1}{M} \sum_{i=1}^{M}\left\|z_{i}-\left(\boldsymbol{R}_{0} \bullet \boldsymbol{y}_{i}+\boldsymbol{T}_{0}\right)\right\|\right\}
$$

In order to solve equation (1), the centroid $\bar{y}$ of the floating point set $\boldsymbol{Y}$ and the centroid $\bar{z}$ of the closet point set $\boldsymbol{Z}$ are first computed by

$$
\left\{\begin{array}{l}
\bar{z}=\frac{1}{M} \sum_{i=1}^{M} z_{i} \\
\bar{y}=\frac{1}{M} \sum_{i=1}^{M} \boldsymbol{y}_{i}
\end{array}\right.
$$

According to equation (2), the cross-covariance matrix $\operatorname{Cov}(\boldsymbol{Z}, \boldsymbol{Y})$ of the point sets $\boldsymbol{Y}$ and $\boldsymbol{Z}$ is computed by

$$
\operatorname{Cov}(\boldsymbol{Z}, \boldsymbol{Y})=\frac{1}{M} \sum_{i=1}^{M}\left[\left(\boldsymbol{y}_{i}-\overline{\boldsymbol{y}}\right)\left(\boldsymbol{z}_{i}-\overline{\boldsymbol{z}}\right)^{T}\right]=\frac{1}{M} \sum_{i=1}^{M}\left[\boldsymbol{y}_{i} \boldsymbol{z}_{i}^{T}\right]-\overline{\boldsymbol{y}} \boldsymbol{z}
$$

The cyclic components of the anti-symmetric matrix $B_{i j}$ of $\operatorname{Cov}(\boldsymbol{Z}, \boldsymbol{Y})$ is defined by

$$
B_{i j}=\left(\operatorname{Cov}(\boldsymbol{Z}, \boldsymbol{Y})-\operatorname{Cov}(\boldsymbol{Z}, \boldsymbol{Y})^{T}\right)_{i j}
$$

$B_{i j}$ is employed to organize the column vector $\boldsymbol{\Omega}$ shown by

$$
\boldsymbol{\Omega}=\left[\begin{array}{lll}
B_{23} & B_{31} & B_{12}
\end{array}\right]^{T}
$$

And now, a symmetric $4 \times 4 \quad \boldsymbol{\Sigma}(\boldsymbol{C o v}(\boldsymbol{Z}, \boldsymbol{Y}))$ is produced by using $\boldsymbol{\Omega}$

$$
\Sigma(\operatorname{Cov}(\boldsymbol{Z}, \boldsymbol{Y}))=\left[\begin{array}{cc}
\operatorname{tr}(\operatorname{Cov}(\boldsymbol{Z}, \boldsymbol{Y})) & \boldsymbol{\Omega}^{T} \\
\boldsymbol{\Omega} & \operatorname{Cov}(\boldsymbol{Z}, \boldsymbol{Y})+\operatorname{Cov}(\boldsymbol{Z}, \boldsymbol{Y})^{T}-\operatorname{tr}(\operatorname{Cov}(\boldsymbol{Z}, \boldsymbol{Y})) \boldsymbol{I}_{3 \times 3}
\end{array}\right]
$$

here $\operatorname{tr}(\bullet)$ means the trace of a matrix, and $\boldsymbol{I}_{3 \times 3}$ is a $3 \times 3$ identity matrix. The unit eigenvector $\boldsymbol{q}=\left[\begin{array}{llll}q_{0} & q_{1} & q_{2} & q_{3}\end{array}\right]$ for $q_{0}^{2}+q_{1}^{2}+q_{2}^{2}+q_{3}^{2}=1$ corresponding to the maximum eigenvalue of the matrix $\Sigma(\boldsymbol{C o v}(\boldsymbol{Y}, \boldsymbol{Z}))$ is regarded as the rotation represented by the unit quaternion. According to $\boldsymbol{q}$, the rotation matrix $\boldsymbol{R}_{0}$ is computed by

$$
\boldsymbol{R}_{0}=\left[\begin{array}{ccc}
q_{0}^{2}+q_{1}^{2}-q_{2}^{2}-q_{3}^{2} & 2\left(q_{1} q_{2}-q_{0} q_{3}\right) & 2\left(q_{1} q_{3}+q_{0} q_{2}\right) \\
2\left(q_{1} q_{2}+q_{0} q_{3}\right) & q_{0}^{2}-q_{1}^{2}+q_{2}^{2}-q_{3}^{2} & 2\left(q_{2} q_{3}-q_{0} q_{1}\right) \\
2\left(q_{1} q_{3}-q_{0} q_{2}\right) & 2\left(q_{2} q_{3}+q_{0} q_{1}\right) & q_{0}^{2}-q_{1}^{2}-q_{2}^{2}+q_{3}^{2}
\end{array}\right]
$$

Then the translation matrix $\boldsymbol{T}_{0}$ is obtained by

$$
\boldsymbol{T}_{0}=\overline{\boldsymbol{z}}-\boldsymbol{R}_{0} \cdot \overline{\boldsymbol{y}}
$$

So far, the rigid transformation parameters are acquired. Repeat the above iterative process until $J\left(\boldsymbol{R}_{0}, \boldsymbol{T}_{0}\right)$ meets a given threshold which denotes the desired registration accuracy or the number of iteration surpasses a given value.

As stated above, ICP proposed by Besl and Mckay is delineated as follows:

Step 1. Initialization: $\varepsilon$ and $k$ express the iteration convergence tolerance and the number of iteration respectively, and we set $k=0$. The initial rotation and translation matrices are initialized by setting $\boldsymbol{R}_{0}^{0}=\left[\begin{array}{lll}1 & 0 & 0 \\ 0 & 1 & 0 \\ 0 & 0 & 1\end{array}\right]$ and $\boldsymbol{T}_{0}^{0}=\left[\begin{array}{lll}0 & 0 & 0\end{array}\right]^{T}$ respectively. Further, the component $\boldsymbol{y}_{j}^{0}$ of the initial floating point set $\boldsymbol{Y}^{0}$ is $\boldsymbol{y}_{j}^{0}=\boldsymbol{R}_{0}^{0} \bullet \boldsymbol{y}_{j}+\boldsymbol{T}_{0}^{0}=\boldsymbol{y}_{j}$ $(j=1,2, \cdots, M)$, namely $\boldsymbol{Y}^{0}=\boldsymbol{Y}$.

Step 2. The closest point set $\boldsymbol{Z}^{k}=\operatorname{Closet}\left(\boldsymbol{Y}^{k}, \boldsymbol{X}\right)$ is calculated. 
Step 3. By applying equations (2)-(8), the rotation and translation matrices $\boldsymbol{R}_{0}^{k+1}$ and $\boldsymbol{T}_{0}^{k+1}$ are computed respectively.

Step 4. Now $\boldsymbol{Y}^{k+1}=\left\{\boldsymbol{y}_{j}^{k+1}, j=1,2, \cdots, M\right\}$ is computed by the following equation

$$
\boldsymbol{y}_{j}^{k+1}=\boldsymbol{R}_{0}^{k+1} \bullet \boldsymbol{y}_{j}+\boldsymbol{T}_{0}^{k+1}
$$

Step 5. According to equation (1) and the object function

$$
\boldsymbol{J}^{k+1}\left(\boldsymbol{R}_{0}^{k+1}, \boldsymbol{T}_{0}^{k+1}\right)=\frac{1}{M} \sum_{i=1}^{M}\left\|z_{i}^{k}-\boldsymbol{y}_{i}^{k+1}\right\|=\frac{1}{M} \sum_{i=1}^{M}\left\|z_{i}^{k}-\left(\boldsymbol{R}_{0}^{k+1} \bullet \boldsymbol{y}_{i}+\boldsymbol{T}_{0}^{k+1}\right)\right\|
$$

Step 6. If $\boldsymbol{J}^{k}\left(\boldsymbol{R}_{0}^{k}, \boldsymbol{T}_{0}^{k}\right)-\boldsymbol{J}^{k+1}\left(\boldsymbol{R}_{0}^{k+1}, \boldsymbol{T}_{0}^{k+1}\right)<\varepsilon$, then the iteration ends, and the optimal parameters $\boldsymbol{R}_{0}^{k+1}$ and $\boldsymbol{T}_{0}^{k+1}$ are acquired; otherwise, go to Step 2 and the iteration continues.

ICP is widely used in the field of image registration. However, it has the following issues. First, it needs to recursively and iteratively explore the closest points, and as a result the computational load is very heavy. Second, whether ICP can successfully extract the optimal parameters for registration strongly depends on the initial rotation and translation values selected. If the initial values are ill-fitted to respond to ICP, then the registration process needs more searching time and even fail to align the images. Third, it is very difficult to automatically select the crucial feature points delineating the image contour in image registration. Furthermore, it easily gets into the local optima.

\section{Medical Image Registration based on Inertia Matrix and Iterative Closest Point}

In order to improve registration efficiency and lessen the possibility of failing to align images, the centroids of the reference and floating images are first obtained, then the rotation angles are acquired by using the coordinate inertia matrices of the reference and floating images, finally the reference and floating point sets are selected by the edge detection operator BSGO.

\subsection{Acquisition of the Centroids of the Medical Images}

For a 2-D discrete function $f(x, y)$, the moment of order $(p+q)$ can be expressed by the following definition $[11,12]$

$$
M_{p, q}=\sum_{x=1}^{M} \sum_{y=1}^{N} x^{p} y^{q} f(x, y) \quad p, q=0,1,2, \cdots
$$

here $(p+q)$ is the order of the moment; $M$ and $N$ represent the numbers of sampling points in space. And well we can define the zeroth as follows[11,12]

$$
M_{0,0}=\sum_{x=1}^{M} \sum_{y=1}^{N} f(x, y)
$$

Further, when $p=1$ and $q=0$, and, $p=0$ and $q=1[11,12]$,

$$
\bar{x}=\frac{M_{1,0}}{M_{0,0}}, \quad \bar{y}=\frac{M_{0,1}}{M_{0,0}}
$$

$(\bar{x}, \bar{y})$ is defined as the centroid coordinates of the object.

When aligning images, we compute the zeroth and first-order moments of reference image $\boldsymbol{R}$ and floating image $\boldsymbol{F}$ respectively by using equations (11) and (12), and then procure the centroids $\left(\overline{x_{R}}, \overline{y_{R}}\right)$ and $\left(\overline{x_{F}}, \overline{y_{F}}\right)$.

Let $(\bar{x}, \bar{y})$ be the object centroids, then we can define the central moment as [11-12]

$$
M_{p, q}^{\prime}=\sum_{x=1}^{M} \sum_{y=1}^{N}(x-\bar{x})^{p}(y-\bar{y})^{q} f(x, y)
$$




\subsection{Calculation of the Rotation Angle of the Medical Image}

The inertia matrix is often applied in image processing fields. The inertia matrix $\boldsymbol{A}$ of the object is expressed by

$$
\boldsymbol{A}=\left[\begin{array}{cc}
M_{0,2}^{\prime} & -M_{1,1}^{\prime} \\
-M_{1,1}^{\prime} & M_{2,0}^{\prime}
\end{array}\right]
$$

The eigenvectors $\boldsymbol{e}_{1}$ and $\boldsymbol{e}_{2}$ of $\boldsymbol{A}$ construct the normalized eigenvector matrix $\boldsymbol{E}$

$$
\boldsymbol{E}=\left[\begin{array}{ll}
\boldsymbol{e}_{1} & \boldsymbol{e}_{2}
\end{array}\right]=\left[\begin{array}{ll}
e_{11} & e_{21} \\
e_{12} & e_{22}
\end{array}\right],
$$

and then we equate $\boldsymbol{E}$ to the rotation matrix

$$
\left[\begin{array}{ll}
e_{11} & e_{21} \\
e_{12} & e_{22}
\end{array}\right]=\left[\begin{array}{cc}
\cos \alpha & -\sin \alpha \\
\sin \alpha & \cos \alpha
\end{array}\right]
$$

where the eignvalues corresponding to $\boldsymbol{E}$ is in decreasing order. From this, the rotation angle $\alpha$ can be obtained by

$$
\alpha=\arctan \left(\frac{e_{12}}{e_{11}}\right) \times \frac{180}{\pi}
$$

or

$$
\alpha=-\arctan \left(\frac{e_{21}}{e_{22}}\right) \times \frac{180}{\pi}
$$

According to the description mentioned above, the method for acquiring the rotation angle of the medical image based on inertia matrix is described as follows:

Step1. Use BSGO to explore the edges of the original image $\boldsymbol{F}$ and produce the binarization edge image $\boldsymbol{B}$;

Step 2. Extract the bounding box of the image $\boldsymbol{B}$, i.e. probe the four boundaries: top, bottom, left, and right ones respectively.

Step 3. Acquire the sub-image $\boldsymbol{F}_{-} \boldsymbol{S} \boldsymbol{u} \boldsymbol{b}$ from the image $\boldsymbol{F}$ according to the bounding box of the image $\boldsymbol{B}$.

Step 4. Compute the zeroth moment $M_{0,0}$, and the first moments $M_{1,0}$ and $M_{0,1}$ of the sub-image $\boldsymbol{F}_{-} \boldsymbol{S} \boldsymbol{S} \boldsymbol{b}$ respectively by using equations (11) and (12).

Step 5. Derive the centroid coordinates $(\bar{x}, \bar{y})$ of the sub-image $\boldsymbol{F}_{-} \boldsymbol{S} \boldsymbol{b} \boldsymbol{b}$ according to equation (13).

Step 6. Unearth the central moments $M_{1,1}^{\prime}, M_{2,0}^{\prime}$ and $M_{0,2}^{\prime}$ of the sub-image $\boldsymbol{F}_{-}$Sub respectively by equation (14).

Step 7. Obtain the eigenvectors $\boldsymbol{e}_{1}$ and $\boldsymbol{e}_{2}$ of $\boldsymbol{A}$ by equation (16).

Step 8. Compute the rotation angle $\alpha$ according to equation (17a) or (17b).

\subsection{IMICP}

In this paper, the initial rotation matrix $\boldsymbol{R}_{0}^{0}$ and translation matrix $\boldsymbol{T}_{0}^{0}$ of ICP are computed by applying the inertia matrix and the centroid coordinates respectively, and the reference and floating point sets are extracted by BSGO. As mentioned previously, medical image registration based on inertia matrix and iterative closest point is described as follows:

Step1. Compute the centroids $\left(\overline{x_{R}}, \overline{y_{R}}\right)$ and $\left(\overline{x_{F}}, \overline{y_{F}}\right)$, and the rotation angles $\alpha_{r}$ and $\alpha_{f}$ of reference image $\boldsymbol{R}$ and the floating image $\boldsymbol{F}$ respectively according to the inertia matrices and the moments of the images.

Step2. Derive the initial values for registration from the following expressions

$$
\Delta x=\bar{x}_{f}-\bar{x}_{r}, \Delta y=\bar{y}_{f}-\bar{y}_{r}, \Delta \alpha=\alpha_{f}-\alpha_{r}
$$


Step3. Use $\Delta x, \Delta y$ and $\Delta \alpha$ as the initial translation and rotation parameters for ICP, namely

$$
\boldsymbol{R}_{0}^{0}=\left[\begin{array}{cc}
\cos (\Delta \alpha) & -\sin (\Delta \alpha) \\
\sin (\Delta \alpha) & \cos (\Delta \alpha)
\end{array}\right], \quad \boldsymbol{T}_{0}^{0}=\left[\begin{array}{ll}
\Delta x & \Delta y
\end{array}\right]^{T}
$$

Step4. Impose BSGO on the reference image $\boldsymbol{R}$ and the floating image $\boldsymbol{F}$ respectively, and then generate the binarized images $\boldsymbol{B}_{R}$ and $\boldsymbol{B}_{F}$ with gray value being 0 or 1 .

Step5. Extract two point sets of coordinates representing all the pixels with gray value being 1 in the images $\boldsymbol{B}_{R}$ and $\boldsymbol{B}_{F}$ respectively as the reference and floating point sets in ICP.

Step6. Perform ICP and derive the final rotation and translation matrices $\boldsymbol{R}_{0}$ and $\boldsymbol{T}_{0}$.

\section{Experiments and Results}

In this paper, all the experimental images are derived from the brain image database found by Retrospective Registration Evaluation Projection, which is affiliated to Vanderbilt University, USA. IMICP is performed in MATLAB 7.1 on PC with an AMD Athlon $3000+2.0 \mathrm{GHz}$ and $512 \mathrm{MB}$ RAM, running Windows XP. In order to validate IMICP with a fast implementation, a high alignment accuracy and a strong reliability, we carry out IMICP and compare the results from ICP. In the following registered images, the red and green landmarks express the results from extracting the edges of the reference and floating images by Canny operator respectively, and the yellow landmarks mark the overlapping region of two registered images.

To order to evaluate the accuracy of the image registration, we use error $\rho_{i}$ [2]

$$
\rho_{i}=\frac{\left|\Delta i-\Delta i_{s}\right|}{\left|\Delta i_{s}\right|} \times 100 \% \quad(i=x, y, \alpha)
$$

where $\Delta i_{s}$ denotes the standard transformation parameter aligning the floating image with the reference image, and $\Delta i$ simplies the actual transformation parameters computed by ICP and IMICP. Besides, we introduce total error $\rho$ [2]

$$
\rho=\sum_{i=\{x, y, \alpha\}} \rho_{i}
$$

\subsection{Mono-modality Medical Image Registration}

In the subsection, we extract No.1 CT and MR_T1 No.1 brain images of the patient_001 as the original experiment images with size being $256 \times 256$ and gray level being 256, shown in Figures 1a and 2a, respectively. Each reference image is transformed into the corresponding floating image according to the transformation parameters in Table 1, shown in Figures 1b, 1c, 2b, and 2c, respectively.

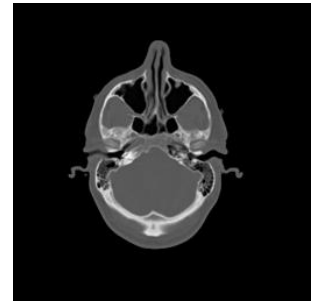

(a)Reference Image

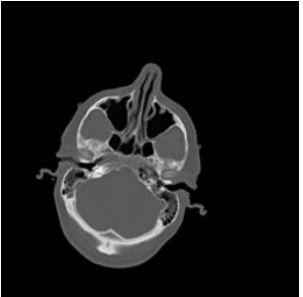

(b)CT1 floating image

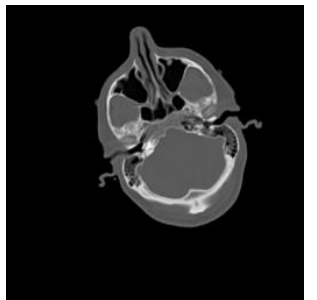

(c)CT2 floating image

Figure 1. CT Experimental Images 


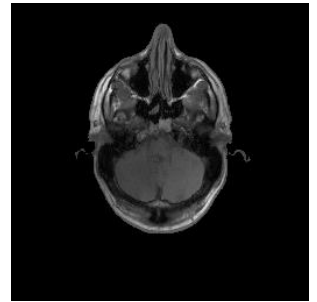

(a)Reference Image

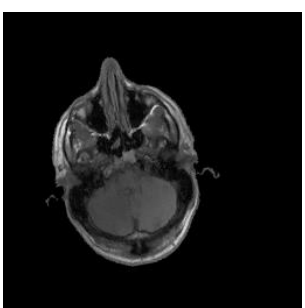

(b)MR1 floating image

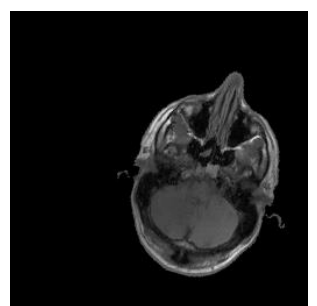

(c)MR2 floating image

Figure 2. MR experimental images

\section{Table 1. Transformation Parameters of Mono-modality Floating Images}

\begin{tabular}{c|c|c|c}
\hline \multirow{2}{*}{ Floating images } & \multicolumn{3}{|c}{ Parameters } \\
\cline { 2 - 4 } & $\Delta x_{s} /$ Pixel & $\Delta y_{s} /$ Pixel & $\Delta \alpha_{s}{ }^{\circ}$ \\
\hline CT1 & 20 & -15 & -12.50 \\
\hline CT2 & -20 & 20 & 20.5 \\
\hline MR1 & 20 & -20 & 10.50 \\
\hline MR2 & 30 & 35 & -15.80 \\
\hline
\end{tabular}

We first use ICP to implement the mono-modality medical image registration, and then use IMICP to register the experimental images. The experimental results are illustrated in Figures 3 and 4 and Table 2.

As shown in the data in Table 2, IMICP is obviously superior to ICP, especially in the registration speed, it attains the overwhelming advantage. With regard to ICP, from the point of registration efficiency, its translation transformation traps in the local optimum and it completely fails to register the images, while IMICP aligns all the images successfully and has a higher registration precision, which is in accordance with the errors shown in Table 2. Therefore, this further verifies the fact that ICP seriously relies on whether the initial rotation and translation matrices of the floating point set can be exactly selected. In the experiments, we find that the initial values derived by the inertia matrices and the centroids are approximate to the transformation parameters in Table 1, which is conducive to significantly reducing the searching time and avoiding getting in the local optimum, and puts a solid foundation for aligning images. In addition, we can know from Table 2, both ICP and IMICP can procure good initial rotation parameters; however, they reap the different initial translation values, which significantly influence on the registration results and the total error. In conclusion, IMICP can be suitable for the monomodality medical image registration.

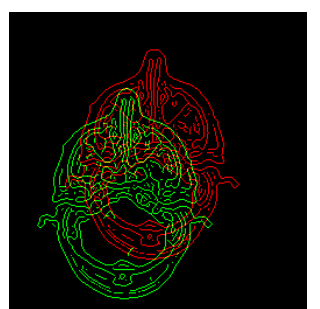

(a) CT1

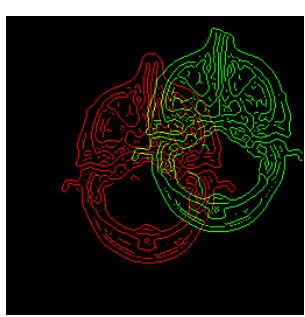

(b) CT2

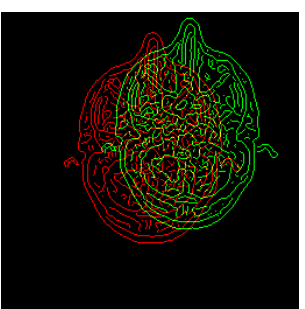

(c) MR1

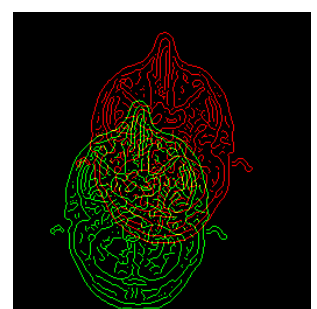

(d) MR2

Figure 3. ICP 


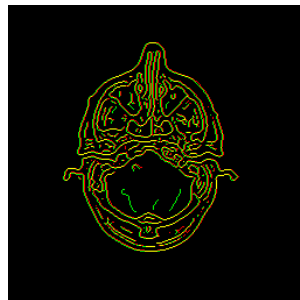

(a) CT1

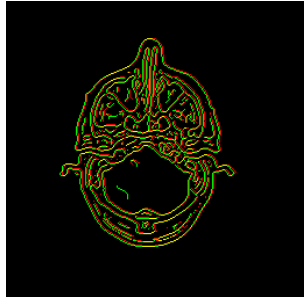

(b) CT2

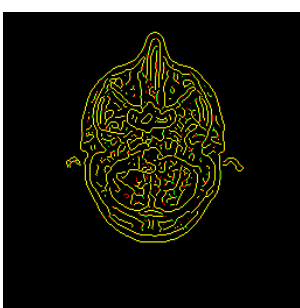

(c) MR1

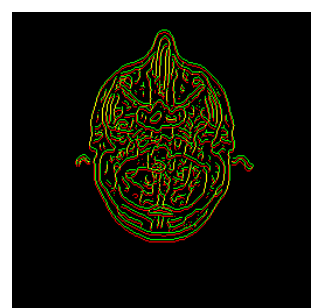

(d) MR2

Figure 4. IMICP

Table 2. Performance of Registering the Mono-modality Images Using ICP and IMICP

\begin{tabular}{c|c|c|c|c|c|c|c|c|c}
\hline \multirow{2}{*}{ Images } & \multirow{2}{*}{$\begin{array}{c}\text { Registration } \\
\text { methods }\end{array}$} & \multicolumn{4}{|c|}{ Parameters } & \multicolumn{4}{c}{ Errors } \\
\cline { 2 - 10 } & $\Delta x /$ Pixel & $\Delta y /$ Pixel & $\Delta \alpha /^{\circ}$ & Time/S & $\rho_{x}$ & $\rho_{y}$ & $\rho_{\alpha}$ & $\rho$ \\
\hline \multirow{3}{*}{ CT1 } & ICP & 8.2225 & -14.5200 & -12.5318 & 15.5000 & 58.8875 & 3.2 & 0.2544 & 62.3419 \\
\cline { 2 - 11 } & IMICP & 19.0375 & -14.9167 & -12.5256 & 3.2572 & 4.8125 & 0.5553 & 0.2048 & 5.5726 \\
\hline \multirow{2}{*}{ CT2 } & ICP & -25.1430 & 27.3503 & 20.5090 & 18.8120 & 25.715 & 36.7515 & 0.0439 & 62.5104 \\
\cline { 2 - 10 } & IMICP & -21.3062 & 20.4461 & 20.5037 & 2.6687 & 6.531 & 2.2305 & 0.018 & 8.7795 \\
\hline \multirow{2}{*}{ MR1 } & ICP & 37.4052 & -49.0081 & 10.5758 & 17.7970 & 87.026 & 145.0405 & 0.7219 & 232.7884 \\
\cline { 2 - 10 } & IMICP & 19.4509 & -20.4034 & 10.3114 & 2.5095 & 2.7455 & 2.017 & 1.7962 & 6.5587 \\
\hline \multirow{3}{*}{ MR2 } & ICP & 20.3724 & 71.9410 & -15.7631 & 22.6100 & 32.092 & 105.5457 & 0.2335 & 137.8713 \\
\cline { 2 - 10 } & IMICP & 31.0838 & 33.9890 & -15.8807 & 3.6321 & 3.6127 & 2.8886 & 0.5108 & 7.0120 \\
\hline
\end{tabular}

\subsection{Multi-modality Medical Image Registration}

In the subsection, we use No.25 CT and MR_T1_rectified and No.24 MR brain images of the training_001 as the experimental images with size being $256 \times 256$ and gray level being 256 . The experimental images are divided into the following two groups. The first is to select CT1 image as the reference one and, MR1 as the floating image, shown in Figure 5. The second is to select MR2 image as the reference one and, CT2 as the floating image, shown in Figure 6. Also, we list the relatively accurate transformation parameters in Table 3, which are taken as $\Delta i_{s}$ in equation (18).

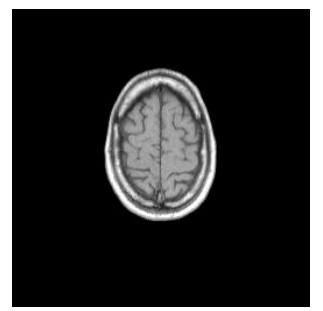

(a)CT1 Reference image

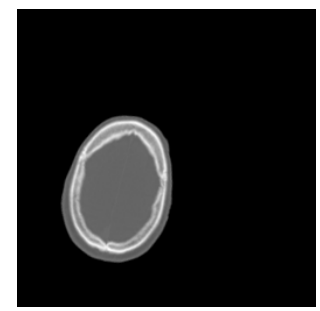

(b)MR1 floating image

Figure 5. The First Group of Images 


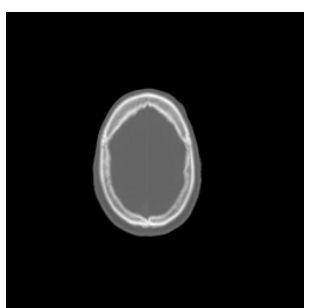

(a)MR2 Reference image

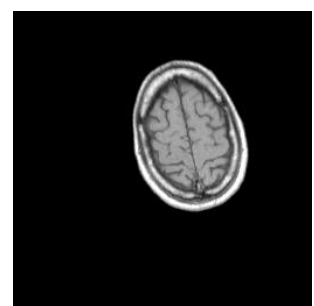

(b)CT2 floating image

Figure 6. The Second Group of Images

Table 3. Transformation Parameters of Multi-modality Floating Images

\begin{tabular}{c|c|c|c}
\hline \multirow{2}{*}{ Floating images } & \multicolumn{3}{|c}{ Parameters } \\
\cline { 2 - 4 } & $\Delta x_{s} /$ Pixel & $\Delta y_{s} /$ Pixel & $\Delta \alpha_{s}{ }^{\circ}$ \\
\hline The first group & 37 & -43 & -14.5 \\
\hline The second group & -24 & 29 & 13.0 \\
\hline
\end{tabular}

Just as before, ICP first and then IMICP are employed to align the multi-modality images respectively. The experimental results are illustrated in Figures 7 and 8 and Table 4.

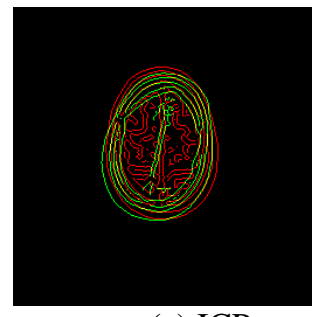

(a) ICP

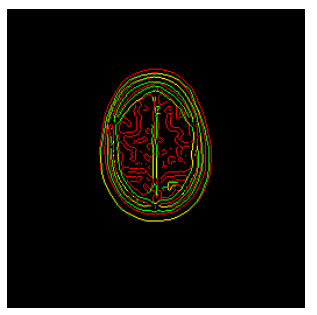

(c) IMICP

Figure 7. Result Figures of Registering Second Group of Images

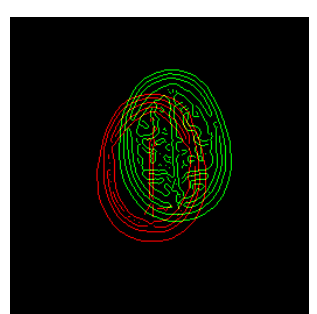

(a) ICP

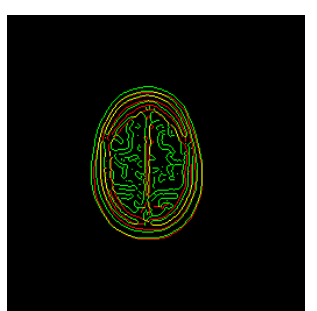

(c) IMICP

Figure 8. Result Figures of Registering First Group of Images

Table. 4 Performance of Registering the Multi-modality Images Using ICP and IMICP

\begin{tabular}{c|c|c|c|c|c|c|c|c|c}
\hline \multirow{2}{*}{ Images } & \multirow{2}{*}{ methods } & \multicolumn{4}{|c|}{ Parameters } & \multicolumn{4}{c}{ Errors } \\
\cline { 3 - 10 } & & $\Delta x /$ Pixel & $\Delta y /$ Pixel & $\Delta \alpha /{ }^{\circ}$ & Time/S & $\rho_{x}$ & $\rho_{y}$ & $\rho_{\alpha}$ & $\rho$ \\
\hline \multirow{2}{*}{ First group } & ICP & 34.0589 & -38.0792 & -1.0312 & 4.6720 & 7.9489 & 11.4437 & 92.8883 & 112.2809 \\
\cline { 2 - 11 } & IMICP & 35.3144 & -42.8279 & -14.5579 & 1.9623 & 4.5557 & 0.4002 & 0.3993 & 5.3552 \\
\hline \multirow{2}{*}{ Second } & ICP & -2.4458 & 11.3784 & 8.3956 & 18.8590 & 89.8092 & 60.7641 & 35.4185 & 185.9918 \\
\hline
\end{tabular}




\begin{tabular}{c|c|c|c|c|c|c|c|c|c}
\hline group & IMICP & -22.5467 & 29.4863 & 13.5024 & 2.2430 & 6.0554 & 1.6769 & 3.8646 & 11.5969 \\
\hline
\end{tabular}

As seen in Table 4, ICP is more time-consuming than IMICP. With respect to the registration accuracy, ICP traps in the local optimum and fails to align the images, while IMICP aligns all the images successfully. Therefore, on the whole, IMICP can be applied for the multi-modality medical image registration.

\section{Conclusions}

In order to tackle the existing problems of aligning the images using ICP, by the inertia matrix and the centroids as the tool for obtaining the initial values for alignment, and BGSO as the method for selecting the feature points, medical image registration based on inertia matrix and iterative closest point is proposed in this paper. The experiments reveal that, IMICP has a simple implementation, a low computational load, a higher registration accuracy, and a remarkable ability that avoids easily getting into the local optimum. IMICP can be well suitable for both mono- and multi-modality image registrations. The study of transplanted IMICP to the non-rigid or 3-D medical image registration will be our next priority direction.

\section{Acknowledgements}

This work is supported by the key Scientific Research Fund of Hunan Provincial Education Department, P.R. China (No.13A064), supported by National Science Foundation of China (No.61403136), supported by the Construct Program of the key Discipline in Hunan University of Arts and Science, and supported by the Doctor Scientific Research Startup Project Foundation of Hunan University of Arts and Science.

\section{References}

[1] M. Nejati and H. Pourghassem, "Multiresolution image registration in digital X-Ray angiography with intensity variation modeling", J Med Syst, vol. 38, no. 1, (2014), pp. 10-18.

[2] M. S. Pan, J. J. Jiang and Q. S. Rong, "A modified medical image registration", Multimed Tools Appl, vol. 70, no. 3, (2014), pp. 1585-1615.

[3] J. B. A. Maintz and M. A. Viergever, "A survey of medical image registration", Med Image Anal, vol. 2, no. 1, (1998), pp. 1-36.

[4] Y. Liu, H. D. Cheng and J. H. Huang, "An effective non-rigid registration approach for ultrasound image based on 'Demons' algorithm”, J Digit Imaging, vol. 26, no. 3, (2013), pp. 521529.

[5] W. Liu and E. Ribeiro, "Incremental variations of image moments for nonlinear image registration", Signal Image Video P, vol. 8, no. 3, (2014), pp. 423-432.

[6] P. Hyunjin, H. Peyton and K. Kristy, "Adaptive registration using local information measures", Med Image Anal, vol. 8, no. 4, (2004), pp. 465-473.

[7] A. Can and C. Stewart, "A feature-based, robust, hierarchical algorithm for registration palm of images of the curved human retina", IEEE T Pattern Anal, vol. 24, no. 3, (2002), pp. 347-363.

[8] P. J. Besl and N. D. Mckay, "A method for registration of 3-D shapes", IEEE T Pattern Anal, vol. 14, no. 2, (1992), pp. 239-256.

[9] K. S. Arun, T. S. Huang and S. D. Blostein, "Least-squares fitting of two 3-D point sets", IEEE T Pattern Anal, vol. 9, no. 5, (1987), pp. 698-700.

[10] S. Kaneko, T. Kondo, and A. Miyamoto, "Robust matching of 3D contours using iterative closest point algorithm improved by M-estimation", Pattern Recogn, vol. 36, no. 9, (2003), pp. 20412047.

[11] M. K. Hu, "Visual pattern recognition by moment invariants", IEEE T Inform Theory, vol. 8, no. 2, (1962), pp. 179-187.

[12] Y. D. Wong, "Matching with invariant moments", Comp Graph Imag Proc, vol. 8, no. 1, (1978), pp. 1624. 


\section{Authors}

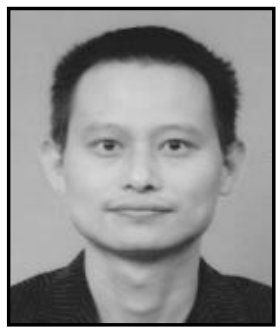

Mei-sen Pan, he was born in 1972 and graduated from Hunan Normal University, China, in 1995. He received the M.S. degree from Huazhong University of Science and Technology, China, in 2005. He obtained the Ph.D. degree from Central South University, China, in 2011. He has published more than 30 papers on journals and conferences. His research interests include biomedical image processing, information fusion and software engineering.

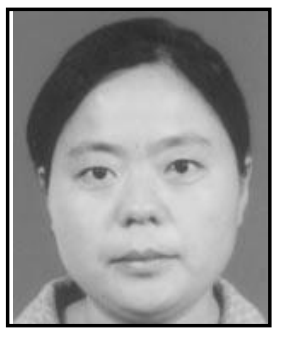

Jian-jun Jiang, she was born in 1971 and graduated from Hunan University of Arts and Science, China, in 2010. She has published 2 papers on journals and conferences. Her research interests include image processing and information retrieves.

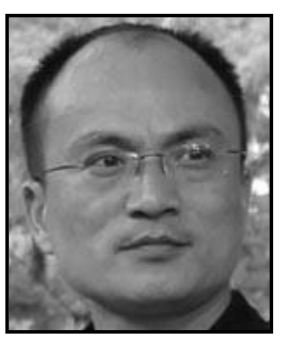

Qiu-sheng Rong, he was born in 1973 and graduated from Central China Normal University, China, in 1996. He received the M.S. degree from Central China Normal University of Science and Technology, China, in 2001. He has published more than 10 papers on journals and conferences. His research interests include data mining and image processing.

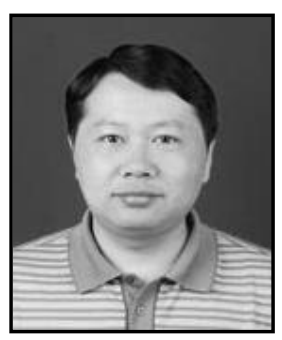

Fen Zhang, he was born in 1972 and graduated from Hunan University, China, in 1996. He received the M.S. degree from Hunan University, China, in 2005. He has published more than 10 papers on journals and conferences. His research interests include image processing and computer graphics. 
International Journal of Signal Processing, Image Processing and Pattern Recognition Vol.8, No.8 (2015) 